\title{
The utility of cardiac stress testing for detection of cardiovascular disease in breast cancer survivors: a systematic review
}

This article was published in the following Dove Press journal:

International Journal of Women's Health

23 January 2015

Number of times this article has been viewed

\author{
Amy A Kirkham' \\ Sean A Virani ${ }^{2}$ \\ Kristin L Campbell ${ }^{1,3}$ \\ 'Rehabilitation Sciences, ${ }^{2}$ Department \\ of Medicine, ${ }^{3}$ Department of Physical \\ Therapy, University of British \\ Columbia, Vancouver, BC, Canada
}

Background: Heart function tests performed with myocardial stress, or "cardiac stress tests", may be beneficial for detection of cardiovascular disease. Women who have been diagnosed with breast cancer are more likely to develop cardiovascular diseases than the general population, in part due to the direct toxic effects of cancer treatment on the cardiovascular system. The aim of this review was to determine the utility of cardiac stress tests for the detection of cardiovascular disease after cardiotoxic breast cancer treatment.

Design: Systematic review.

Methods: Medline and Embase were searched for studies utilizing heart function tests in breast cancer survivors. Studies utilizing a cardiac stress test and a heart function test performed at rest were included to determine whether stress provided added benefit to identifying cardiac abnormalities that were undetected at rest within each study.

Results: Fourteen studies were identified. Overall, there was a benefit to utilizing stress tests over tests at rest in identifying evidence of cardiovascular disease in five studies, a possible benefit in five studies, and no benefit in four studies. The most common type of stress test was myocardial perfusion imaging, where reversible perfusion defects were detected under stress in individuals who had no defects at rest, in five of seven studies of long-term follow-up. Two studies demonstrated the benefit of stress echocardiography over resting echocardiography for detecting left ventricular dysfunction in anthracycline-treated breast cancer survivors. There was no benefit of stress cardiac magnetic resonance imaging in one study. Two studies showed a potential benefit of stress electrocardiography, whereas three others did not.

Conclusion: The use of cardiac stress with myocardial perfusion imaging and echocardiography may provide added benefit to tests performed at rest for detection of cardiovascular disease in breast cancer survivors, and merits further research.

Keywords: heart diseases, heart function tests, exercise test, breast neoplasms, review

\section{Introduction}

Adjuvant treatment for breast cancer, particularly some chemotherapy regimens and leftsided radiotherapy, are well-recognized to cause direct adverse effects on the cardiovascular system. ${ }^{1}$ Thanks to progressively effective antineoplastic therapies, women diagnosed with breast cancer are increasingly living longer, which increases the age-related risk of cardiovascular disease, independent of breast cancer treatment. ${ }^{2}$ An important determinant of the increased risk of cardiovascular disease with age in women is the occurrence of menopause ${ }^{3}$ chemotherapy for breast cancer will induce menopause in 21 to $100 \%$ of women, depending on age. ${ }^{4}$ In light of this combination of risk factors, it is not surprising then that women who have been treated for breast cancer are more likely to die of cardiovascular disease than women who have not had breast cancer., ${ }^{5,6}$
Correspondence: Kristin L Campbell Department of Physical Therapy, University of British Columbia, 212, 2177 Wesbrook Mall, Vancouver,

BC, Canada V6T IZ3

Tel +l 6048274704

Fax + I 6048221870

Email kristin.campbell@ubc.ca 
The cardiovascular toxicity of breast cancer treatment can occur as an early effect, within the first year of treatment, or as a late effect, occurring years to decades after treatment. ${ }^{7,8}$ In both instances, individuals may often be asymptomatic before progression to overt disease. ${ }^{9}$ Therefore, there is great interest in sensitive heart function tests for detection of occult cardiovascular disease, both during treatment and in long-term follow-up in this population. The utility of resting echocardiography, resting electrocardiography (ECG), resting nuclear imaging techniques, and serum cardiac biomarkers for early detection of cardiovascular disease in cancer survivors has been reviewed extensively. ${ }^{10-14}$ Although these diagnostic techniques do appear to have some sensitivity for cancer treatment-induced cardiac abnormalities, cardiac stress tests could be an effective technique for uncovering occult cardiovascular disease related to breast cancer treatment, including coronary artery disease and left ventricular dysfunction.

The human body is a complex, adaptive system with incredible capacity for compensation. Compensatory patterns may maintain homeostasis at rest; however, the introduction of stress challenges the stability of the system, and occult dysfunction may be unmasked. For example, the myocardium may be adequately perfused at rest, despite sub-total occlusion of a major epicardial coronary artery. ${ }^{15}$ However, with the heightened metabolic demands of an increased physical workload, and corresponding myocardial blood flow requirements, there is insufficient coronary reserve, resulting in diminished myocardial perfusion and ischemia. ${ }^{15}$ Similarly, stress may also uncover systolic dysfunction that may not be apparent at rest. Cardiac stress testing or, more generally, heart function tests performed under myocardial stress, may reveal occult coronary artery disease or left ventricular dysfunction that is not apparent with heart function tests performed at rest, due to compensatory mechanisms. In addition to exercise, a number of pharmacological agents or, less commonly, a cold pressor test may be used to impose myocardial stress.

The purpose of this systematic review was to determine the utility of myocardial stress in combination with heart function tests, or "cardiac stress tests", in detection of evidence of cardiovascular disease among breast cancer survivors. To our knowledge, this is the first review to compare cardiac stress tests against tests performed at rest, for the identification of cardiovascular disease among a population treated for cancer.

\section{Methods}

\section{Search strategy}

The search strategy was formulated in consultation with a research librarian. Medline (1946 to present) and Embase
(1947 to present) were searched up to 21 November 2013. The Ovid interface was used to search the Medline and Embase databases. The detailed search for Medline is provided in Table 1. The general search strategy involved (breast cancer) AND (keywords/MeSH [medical subject heading] terms related to cardiac imaging/heart function tests) AND (keywords/MeSH terms related to cardiotoxicity OR cardiotoxic cancer treatments OR related cardiac issues). The search strategy included search terms for all types of cardiac imaging techniques and heart function tests, with or without stress. The search was limited to human studies and English-language papers published in peer-reviewed journals. Experimental, observational, prospective, and retrospective study designs were included; reviews, editorials, letters to the editor, and comments were excluded. Other inclusion criteria involved: study participants diagnosed with any stage of breast cancer; any age range; at least ten study participants; and count data of heart function test results, or statistical analysis of results between a group who received cardiotoxic treatment and a group who did not.

No studies involving breast cancer survivors were identified that compared cardiac stress tests to the gold standard for diagnosis of cardiotoxicity, endomyocardial biopsy, likely because this technique is invasive and rarely performed. Therefore, in lieu of a reference standard, an alternative technique for assessing the utility of cardiac stress tests in breast cancer survivors was required. An inclusion criterion was added that studies must include a cardiac stress test and at least one heart function test performed at rest. The outcome of the cardiac stress test was compared to that of a test at rest to determine whether the use of stress identified evidence of occult cardiovascular disease that was not detected at rest.

The reference lists of all studies meeting inclusion criteria and related studies were hand searched for additional relevant publications. Studies with relevant titles but no abstract were included in full text review. Studies with abstracts that reported the use of two or more heart function tests, without enough information to determine whether they were used under resting conditions, were included in full text review. Upon full text review, the methods section of these articles was searched to determine the use of stress with at least one of the tests, in addition to at least one test performed at rest, for comparison. If the methods section did not describe the use of stress, it was assumed that all tests were performed at rest. Authors were not contacted to acquire additional information or data.

\section{Data collection}

The following data were extracted from each study: sample size; breast cancer treatments received by study participants; 


\section{Table I Ovid Medline search strategy}

I breast neoplasms/ or exp carcinoma, ductal, breast/

2 breast cancer.ti,ab.

$3 \quad$ I or 2

4 exp cardiac imaging techniques/ or ultrasonography/ or echocardiography/ or echocardiography, doppler/ or exp echocardiography, stress/ or echocardiography, three-dimensional/ or ultrasonography, doppler/ or exp echocardiography, doppler/ or ultrasonography, doppler, duplex/ or ultrasonography, doppler, pulsed/ or diagnostic techniques, cardiovascular/ or heart function tests/ or cardiac output/ or cardiography, impedance/ or echocardiography, stress/ or electrocardiography/ or exercise test/ or heart auscultation/ or heart catheterization/ or magnetocardiography/ or myocardial perfusion imaging/ or oximetry/ or radionuclide ventriculography/ or plethysmography/ or biological assay/ or exp ergometry/ or exp exercise test/

5 cardiac evaluation.ti,ab.

6 card* follow-up.ti.

7 echocardio*.ti,ab.

8 exercise echocardiography.ti,ab.

9 exp dobutamine/du [diagnostic use]

10 dobutamine.ti,ab.

II doppler.ti.

12 radionuclide.ti.

13 or $/ 4-12$

14 exp magnetic resonance imaging/

$15 \quad 13$ and 14

$16 \quad 15$ not (screening or mammogra*).ti.

$17 \quad 13$ or 16

18 exp anthracyclines/ae, po, to [adverse effects, poisoning, toxicity]

19 (anthracyclines or anthracycline antibiotics or doxorubicin or adriamycin or epirubicin or idarubicin or daunorubicin or rubidomycin or daunoxome or myocet or caelyx or doxil).ti,ab.

20 fluorouracil.ti,ab.

$21 \quad$ cyclophosphamide.ti,ab.

22 (herceptin or trastuzumab).ti,ab.

23 exp antineoplastic protocols/ or exp chemotherapy, adjuvant/ or exp radiotherapy, adjuvant/ or exp molecular targeted therapy/

24 chemotherapy-induced.ti,ab.

25 exp radiotherapy, adjuvant/ae [adverse effects]

26 radiation.ti,ab.

27 radiation-induced.ti,ab.

28 cancer treatment.ti.

29 cancer therapy.ti.

30 exp antibodies, monoclonal/ae, po, to [adverse effects, poisoning, toxicity]

3I or/l8-30

32 exp drug toxicity/

33 toxicity tests/ or acute toxicity tests/ or cytotoxicity tests, immunologic/ or toxicity tests, chronic/

34 heart diseases/ or arrhythmias, cardiac/ or cardiac output, high/ or cardiac output, low/ or arrhythmogenic right ventricular dysplasia/ or cardiomyopathy, dilated/ or cardiomyopathy, hypertrophic/ or cardiomyopathy, restrictive/ or endomyocardial fibrosis/ or myocarditis/ or heart failure/ or exp myocardial ischemia/ or acute coronary syndrome/ or angina pectoris/ or coronary disease/ or myocardial infarction/ or pericarditis, constrictive/ or exp ventricular dysfunction/

35 heart/de, re, to

36 cardiac dysfunction.ti,ab.

37 cardiomyopath*.ti,ab.

38 cardiotoxic*.ti,ab.

39 cardiac toxic*.ti,ab.

40 heart damage.ti,ab.

4I heart failure/ci

42 cardiovascular injury.ti,ab.

43 (cardiac adj3 injury).ti,ab.

44 or $/ 32-43$

453 3 or 44

$46 \quad 3$ and 17 and 45

$47 \quad 46$ not (animal/ not human/)

$48 \quad 48$ limit 47 to english language

Abbreviations: ab, abstract; ae, adverse events; de, drug effects; du, diagnostic use; exp, explode; po, poisoning; re, radiation effects; ti, title; to, toxicity; *, truncation that expands a search term to include all forms of a root word. 
dosage and timing of delivery of treatment; timing of heart function tests with respect to treatment receipt; type of each test used with stress; type of stress used; type of each test used at rest; parameters of each test measured; and results for each parameter.

\section{Determination of utility of cardiac stress tests in detection of cardiovascular disease}

The findings of the cardiac stress tests were compared to the tests at rest to determine the utility of the stress test within each individual study. Studies were labeled as "no benefit", "possible benefit", or "benefit", based on whether the cardiac stress tests identified evidence of cardiovascular disease in the study participants that was not identified by the heart function tests performed at rest in that study. Where applicable, the reported thresholds for determining cardiovascular dysfunction (eg, LVEF [left ventricular ejection fraction] lower limits of normal) from each individual paper were used. In other cases, the identification of abnormalities was taken as evidence of occult cardiovascular disease (eg, identification of perfusion defects with myocardial perfusion imaging). The study was labeled "no benefit" if: 1) the cardiac stress test did not identify additional study participants with evidence of cardiovascular disease who were not identified with tests at rest, or 2) the cardiac stress tests and the tests at rest were either both statistically different or both not different between treated and non-treated groups. The study was labeled "benefit" if: 1) the cardiac stress test identified study participants with evidence of cardiovascular disease who were not identified by the techniques at rest, or 2) there was a statistically significant difference in cardiac function for the cardiac stress tests, but not for the tests performed at rest. The label "possible benefit" was assigned to studies where the cardiac stress test identified evidence of cardiovascular disease, but it could not be determined from the reported data whether the cases identified with tests performed at rest occurred in the same study participants as the cases identified with stress tests.

\section{Results}

The process for selection of papers for this review is outlined in Figure 1. Fourteen studies ${ }^{16-29}$ met the inclusion criteria for the review and are summarized in Table 2.

\section{Utility of cardiac stress tests}

Overall, five studies ${ }^{16,22,23,25,26}$ showed a benefit, five ${ }^{18,19,24,27,28}$ showed possible benefit, and four ${ }^{17,20,21,29}$ showed no benefit to utilizing cardiac stress tests in identifying evidence of cardiovascular disease in breast cancer survivors.

\section{Cardiac stress test types}

Heart function tests that were performed with stress in the selected studies were scintigraphy $(n=5),{ }^{17,19,20,28,29}$ single photon emission computed tomography (SPECT) $(n=4),{ }^{18,22,24,25}$ echocardiography $(\mathrm{n}=2),{ }^{16,23}$ cardiac magnetic resonance imaging (cMRI) $(n=1),{ }^{21}$ nuclear ventriculography $(n=1),{ }^{26}$ and ECG $(n=5) .{ }^{17,19,20,22,27}$ The utility of stress among each technique is shown in Figure 2A.

Stress myocardial perfusion imaging techniques (ie, scintigraphy or SPECT) were the most commonly used type of cardiac stress test, used in nine studies. The timing and treatment type distributions (ie, chemotherapy, radiotherapy, or trastuzumab) of the effects are shown in Figure 2B. The most common use of myocardial perfusion imaging was for long-term follow-up to radiotherapy, in seven studies, showing a benefit ${ }^{22,25}$ or possible benefit ${ }^{18,19,24}$ in five of these. Stress echocardiography was used in two studies that both demonstrated a benefit in detecting anthracycline-induced left ventricular dysfunction. ${ }^{16,23}$ The timing of imaging in one study was before, during, and after (ie, before each cycle, then $2,4,7$, and $12-18$ months after) chemotherapy, ${ }^{16}$ while, in the other study, imaging was a one-time assessment in longterm follow-up to chemotherapy treatment (mean follow-up of 34 months). ${ }^{23}$ Stress ECG was utilized in five studies: one demonstrated a benefit, ${ }^{22}$ although the benefit in this study was attributed to stress SPECT, not ECG; two demonstrated possible benefit, ${ }^{19,27}$ and two no benefit. ${ }^{17,20}$ Stress ventriculography was used in one study and demonstrated a benefit, compared to testing performed at rest; ${ }^{26}$ while stress cMRI was also used in one study and failed to show a benefit, relative to resting imaging. ${ }^{21}$

\section{Breast cancer treatments}

The adjuvant breast cancer treatments included in the studies were chemotherapy, radiotherapy, and a targeted, humanized, monoclonal antibody (trastuzumab). The overall utility of cardiac stress tests among the different treatment types is displayed in Figure 2C. Cardiac stress tests appear to be most beneficial for detecting cardiovascular disease following chemotherapy, or chemotherapy and radiotherapy combined treatment.

\section{Method of inducing myocardial stress}

Exercise was used to induce stress in eight studies, ${ }^{17-20,23,25,27,29}$ pharmacological agents were used in five studies ${ }^{16,21,22,24,25}$ 


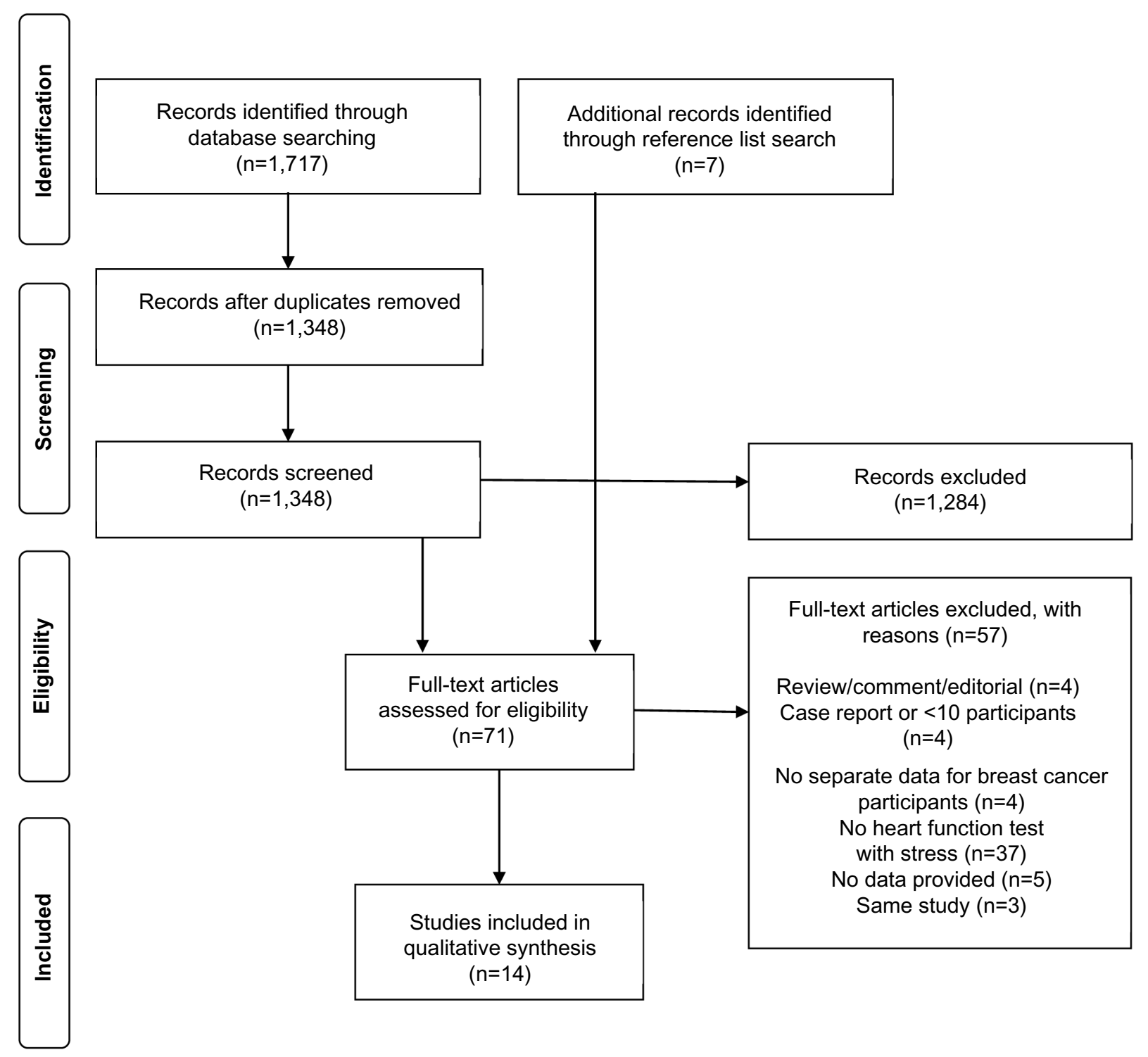

Figure I Preferred Reporting Items for Systematic Reviews and Meta-Analyses (PRISMA) flow diagram of study selection.

and the cold pressor test in one study. ${ }^{26}$ The utility of the use of exercise, pharmacological agents, or the cold pressor test to induce myocardial stress within the studies is displayed in Figure 2D. Exercise and pharmacological agents demonstrated a benefit or possible benefit in $63 \%$ and $80 \%$ of the studies they were used in to induce stress, respectively. The one study to use the cold-pressor test to induce stress demonstrated a benefit. No adverse reactions to stress were reported.

\section{Discussion}

This review demonstrated that cardiac stress testing may be beneficial in detecting evidence of cardiovascular disease in breast cancer survivors that was missed by heart function tests performed at rest. The primary findings of this review are that, in general, cardiac stress tests appear to be most beneficial in chemotherapy-treated populations (with or without radiotherapy), while, specifically, stress myocardial perfusion imaging appears to be beneficial for long-term follow-up to radiotherapy, and stress echocardiography appears to be beneficial in detecting left ventricular dysfunction following anthracycline treatment.

With an approximate 5 -year survival rate of $80 \%$ or greater for all stages of breast cancer combined in North America and Europe,${ }^{30-32}$ cancer is beginning to be considered a manageable disease. ${ }^{33}$ Short and long-term cardiac effects 


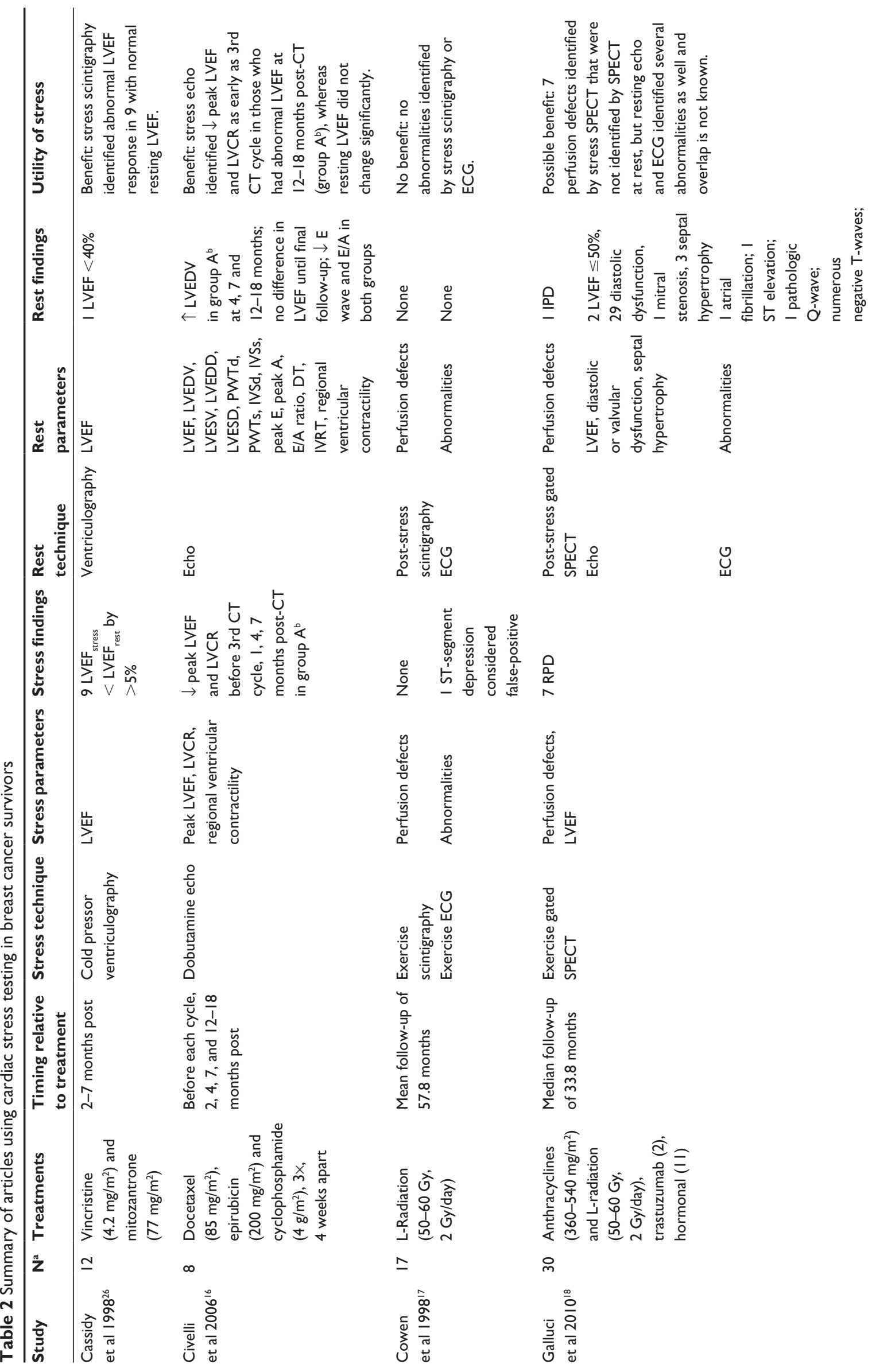



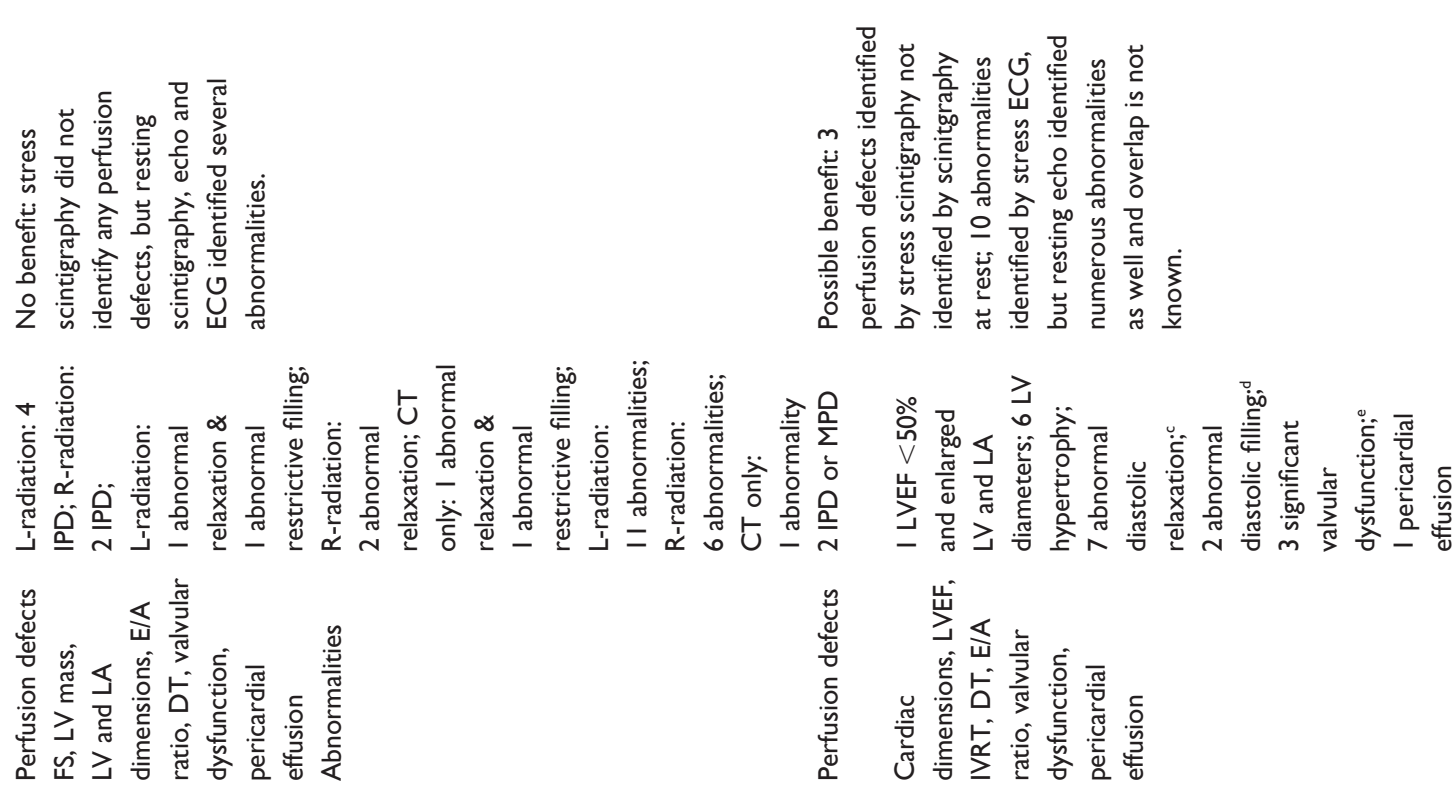

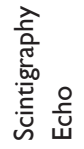

U

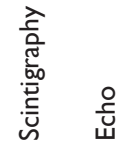

$\stackrel{0}{\text { ํํํ }}$

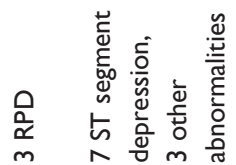

$\frac{0}{0}$
$\frac{0}{0}$
0
$\frac{0}{0}$
$\frac{0}{0}$
$\frac{1}{0}$
0

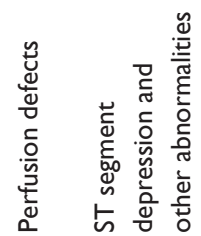

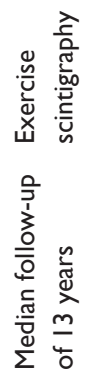

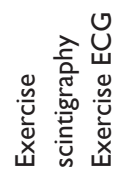

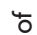

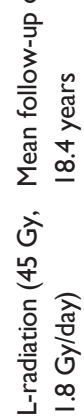

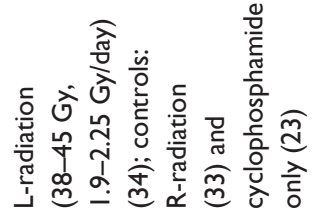

成

号

告

m

우

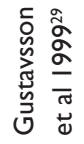

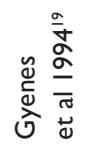




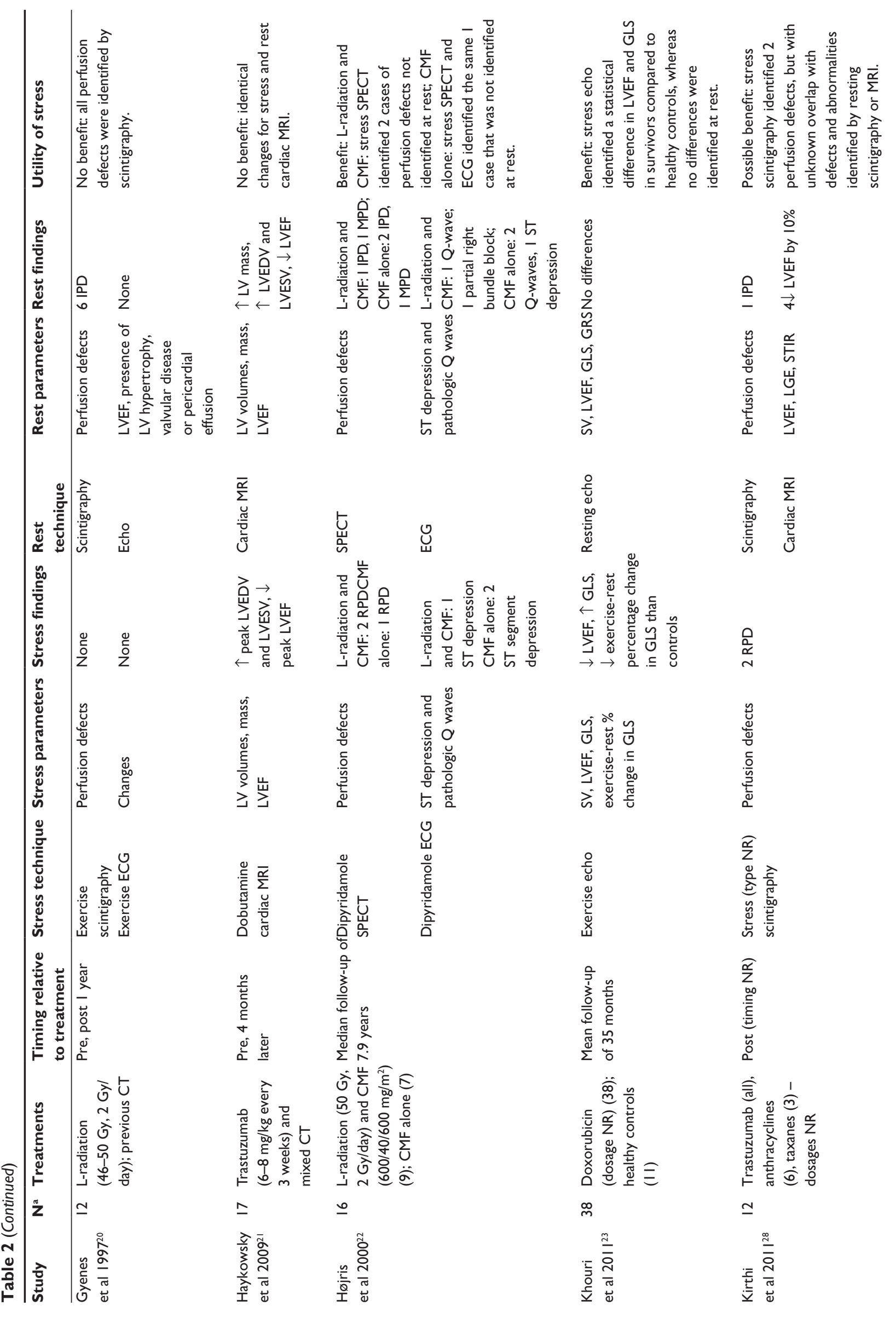




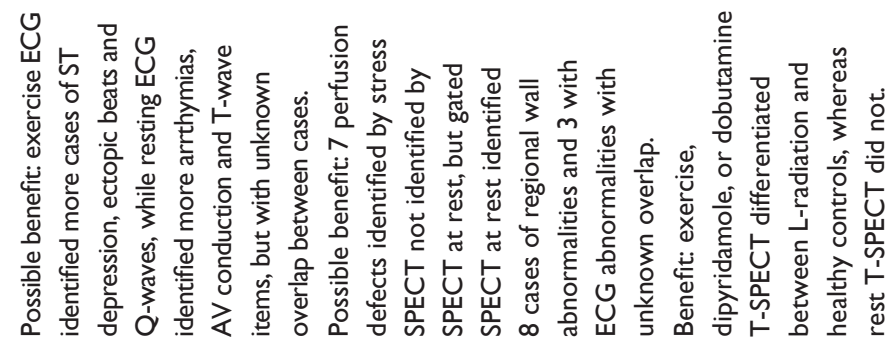
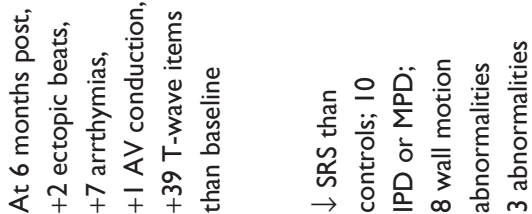

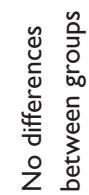

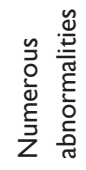

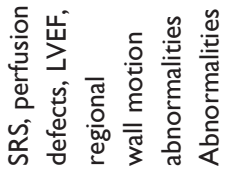

巡

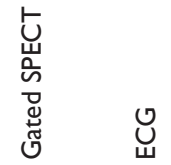

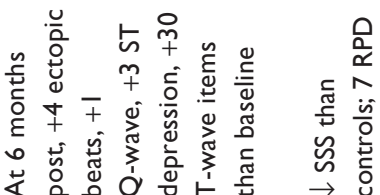

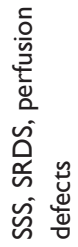

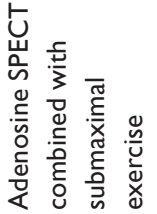

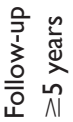

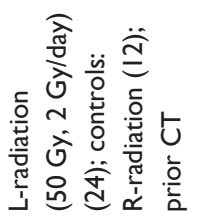

产密
ป

密 $\mathscr{\infty}$

总

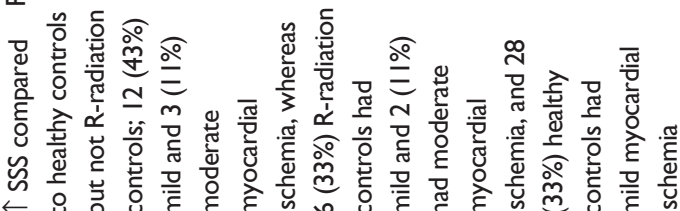

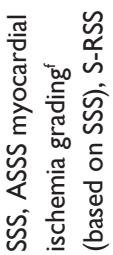

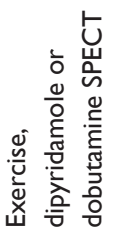

唔

⿳亠口了阝

응

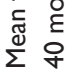

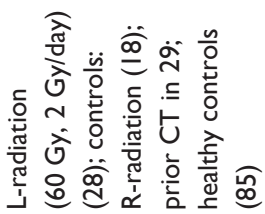

$\infty$

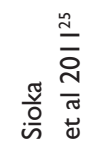

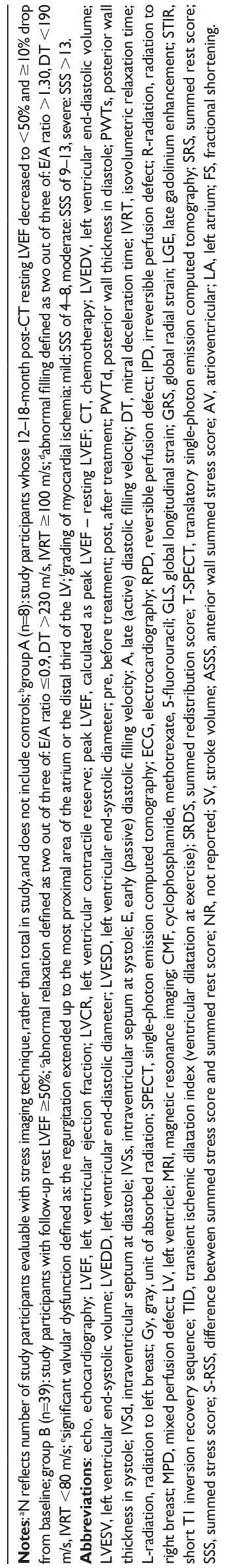


A

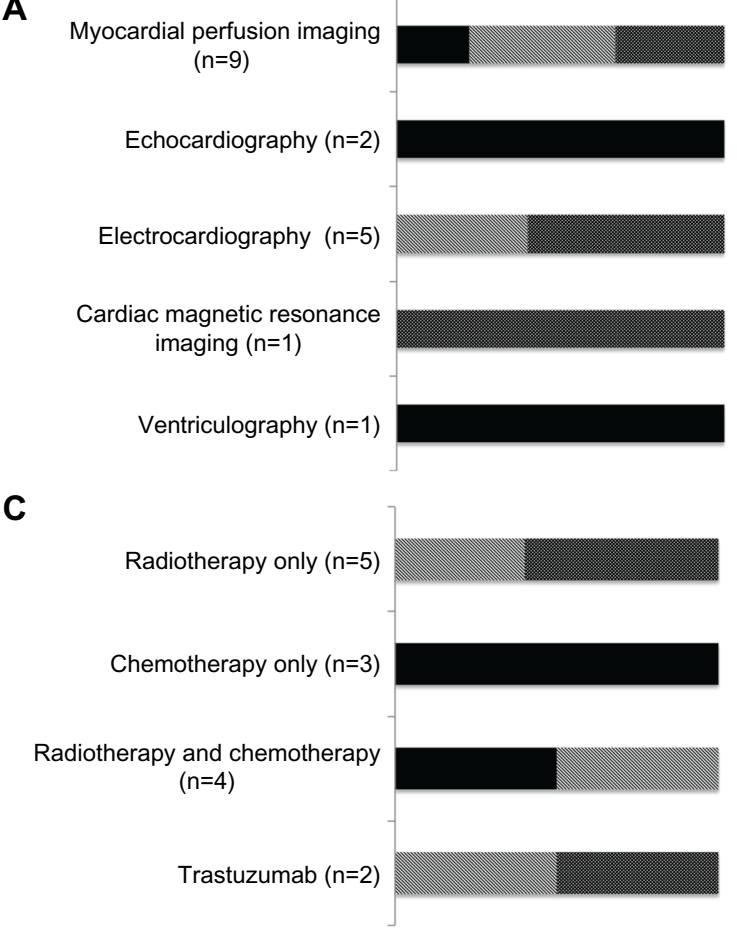

B

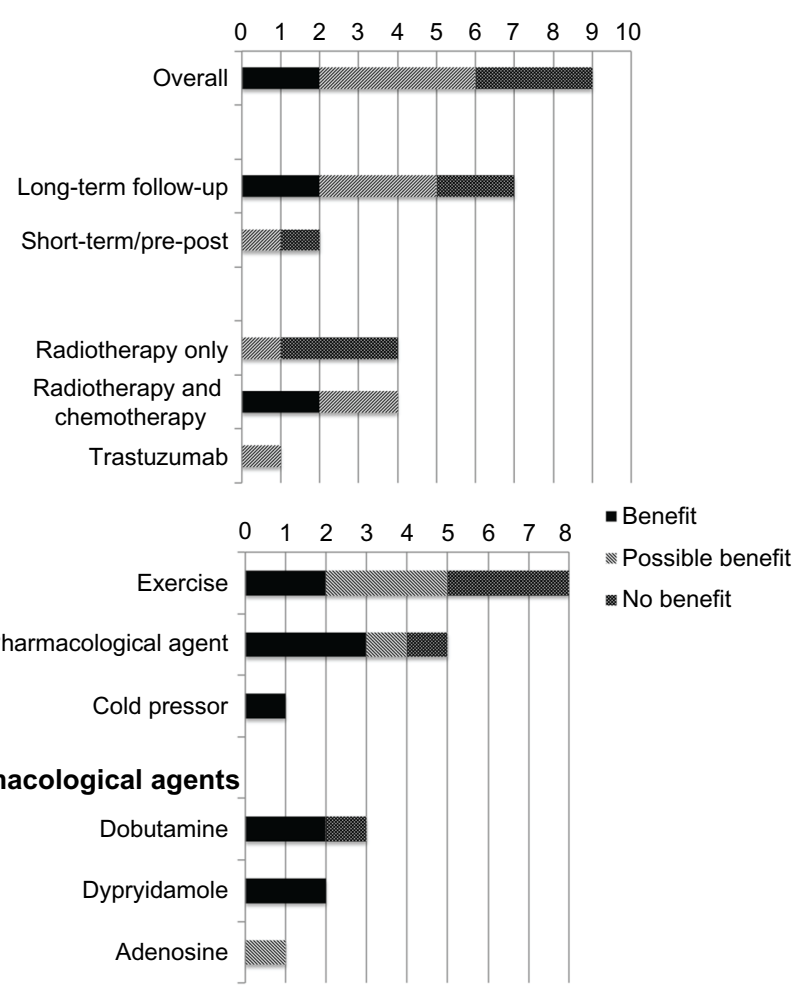

Figure 2 Distribution of utility (ie, "benefit", "possible benefit", or "no benefit”) of stress.

Notes: (A) Distribution of utility among different techniques. (B) Timing of imaging and types of treatment among studies utilizing myocardial perfusion imaging techniques. (C) Distribution of utility among different breast cancer treatments. (D) Utility of type of stress. Note that one study used exercise, dobutamine, or dipryidamole, and is counted in each category. ${ }^{25}$

of cancer treatment are significant determinants of all-cause mortality rates for cancer survivors. ${ }^{9}$ As such, identification of the optimal screening strategies for cardiovascular effects of cancer therapies is a high priority. ${ }^{9}$

Heart function tests, in combination with exercise or pharmacological stress, are commonly used for diagnostic and prognostic cardiac care, ${ }^{34,35}$ and have been used to a limited extent for long-term follow-up in childhood cancer survivors treated with chemotherapy. ${ }^{36-43}$ In this review, two studies ${ }^{16,23}$ demonstrated the benefit of parameters of global systolic function measured via stress echocardiography in identifying left ventricular dysfunction in breast cancer survivors who have received a cardiotoxic systemic treatment. Khouri et $\mathrm{al}^{23}$ demonstrated that the change in global longitudinal strain from rest to peak exercise was significantly different between anthracycline-treated breast cancer survivors and healthy controls. In addition to long-term cardiac effects, anthracyclines are also associated with acute or early cardiac effects, defined as occurring between treatment initiation and 1 year later. ${ }^{8}$ Early identification of left ventricular dysfunction is critical, as it allows for earlier intervention in the form of dose reduction or initiation of therapeutic measures. Civelli et $\mathrm{al}^{16}$ monitored patients for early cardiotoxicity during ongoing anthracycline chemotherapy treatment, and demonstrated the ability of dobutamine echocardiography to identify subclinical left ventricular dysfunction with a reduction in left ventricular contractile reserve (LVCR) (stress-rest LVEF) before the third chemotherapy cycle. This study also demonstrated the predictive value of LVCR for traditional diagnostic parameters of cardiotoxicity (resting LVEF decrease to $<50 \%$ and $\geq 10 \%$ drop from baseline) at $12-18$ months post-chemotherapy.

Radiation to the breast can cause ultrastructural damage to myocardial capillaries and valves, atherosclerosis in coronary arteries, valvular abnormalities, and myocardial fibrosis leading to diastolic and systolic dysfunction, pericardial inflammation, or effusion. ${ }^{44-47}$ There is a 1.27 excess rate ratio of heart disease in women treated with radiation for breast cancer relative to those who were not. ${ }^{48}$ Mortality from coronary artery disease and risk of cardiovascular disease has decreased for breast cancer survivors diagnosed after $1980,{ }^{6,49}$ although more follow-up data is need to determine long-term risk associated with more modern treatment techniques. The cardiac injury associated with radiotherapy for breast cancer is typically a "late effect", occurring years to decades after treatment. ${ }^{7}$ Long-term cardiac follow-up is 
suggested for breast cancer survivors who receive radiotherapy. ${ }^{50}$ In this review, myocardial perfusion imaging was used in several studies of long-term follow-up to radiotherapy. To perform this type of imaging, a radioactive tracer substance is injected, and its accumulation in the myocardium, as detected by a gamma camera, is considered proportional to the myocardial blood flow. ${ }^{15}$ A perfusion defect can be identified on the image produced as an area of decreased perfusion or injured heart tissue resulting from a diseased coronary artery. A perfusion defect that is detected at rest and does not change during stress imaging is considered fixed or irreversible, and suggests non-viable myocardium; while a perfusion defect that is absent at rest but detected during stress is considered reversible, and indicates ischemic myocardium. ${ }^{51}$ Five studies ${ }^{18,19,22,24,28}$ in this review identified reversible defects with stress tests that would not have been discovered with tests performed at rest alone in breast cancer survivors treated with left-sided radiotherapy and other systemic treatments. This suggests that stress myocardial perfusion imaging could be an effective method of long-term follow-up for detection of coronary artery disease in radiation-treated breast cancer survivors. Gyenes et $\mathrm{al}^{20}$ reported several fixed perfusion defects, but no reversible defects at 1 year post-radiotherapy. An incidence of fixed perfusion defects of $60 \%$ has been reported 6 months following radiotherapy for left-sided breast cancer. ${ }^{52}$ It may be that the utility of stress myocardial perfusion imaging for screening purposes is limited to long-term follow-up only.

It is unclear from this review whether stress ECG is beneficial for identifying cardiovascular disease in breast cancer survivors. Two studies demonstrated possible benefit. In one of these, ${ }^{27}$ ECG was used under stress and at rest, and stress did appear to better detect specific abnormalities, namely ectopic beats and, not surprisingly, minor ST depressions that developed 6 months after radiotherapy to the sternum. However, numerous arrthymias and more T-wave items were detected only at rest and there was unknown overlap between abnormalities detected under each condition. A similar case of unknown overlap between abnormalities detected with stress ECG and resting echocardiography occurred in the other study of long-term follow-up. ${ }^{19}$ The lack of ability to detect occult cardiovascular disease may be attributed to the temporally late occurrence of electrocardiographic changes in the cascade of occurrences in the development of ischemia. ${ }^{53}$

Baseline and periodic monitoring of cardiac function is recommended in individuals treated with trastuzumab. ${ }^{50}$ Haykowsky et $\mathrm{al}^{21}$ used cMRI, at rest and with dobutamine stress, at baseline and 4 months into trastuzumab treatment.
This study did not demonstrate the utility of cardiac stress, as the cMRI findings were the same at rest and with stress. However, left ventricular contractile reserve was not reported, which is a more sensitive measure of early left ventricular dysfunction, as evidenced by Civelli et al. ${ }^{16} \mathrm{Kirthi}$ et $\mathrm{al}^{28}$ used scintigraphy at rest and with stress, in addition to resting cMRI, following trastuzumab treatment. One additional case of cardiovascular dysfunction was identified with stress scintigraphy, compared against scintigraphy at rest, demonstrating a benefit for this technique alone; however, cMRI at rest also identified four cases of cardiovascular dysfunction. $^{28}$

Myocardial stress can be induced by exercise, a number of pharmacological agents, or a cold pressor test. Pharmacological-induced stress minimizes factors such as movement artifact that may degrade cardiac imaging quality and decrease accuracy, but incurs the additional cost of the pharmacological agent. ${ }^{53}$ The cold pressor test would likely carry similar advantages, but does not incur additional costs. However, the clinical significance of a cold pressor-induced change in cardiovascular function is unclear. Dynamic exercise tends to be the preferred method to induce myocardial stress, because it is less expensive, perceived to be safer, and the equipment (ie, cycle ergometer or treadmill) is widely available in clinical settings. ${ }^{54}$ Exercise stress also provides additional ancillary prognostic, diagnostic, and therapeutic information, such as peak oxygen consumption, heart rate, and blood pressure response, and variables required for exercise prescription. However, the inability to reach maximal exercise capacity will limit the sensitivity of stress tests, in which case pharmacological stress is a suitable alternative. ${ }^{54}$

There is additional cost involved in cardiac stress tests with either exercise or pharmacological stress. For example, stress echocardiography is approximately double the cost of resting echocardiography, ${ }^{55}$ but is reported to potentially enhance overall cost-effectiveness for diagnosis and management of suspected cardiovascular disease. ${ }^{56}$ Stress myocardial perfusion imaging is often performed in addition to imaging at rest; however, the use of stress myocardial perfusion imaging alone would decrease the cost, radiation exposure, and time of testing, and is not associated with differences in prognosis or mortality outcomes. ${ }^{57}$

In ten of the 14 studies in this review, evidence of cardiovascular disease was missed by heart function tests performed at rest, but detectable with cardiac stress tests. This information is relevant to those involved in the treatment and care of breast cancer survivors, including family physicians, medical and radiation oncologists, and cardiologists. 
Lack of change in the most common oncological clinical parameter of cardiac function, resting $\mathrm{LVEF}$, is often equated to a lack of cardiotoxicity, despite convincing evidence in the literature that substantial myocyte damage can occur before a change in LVEF results. ${ }^{58}$ However, when exercise stress is used to evaluate change in LVEF, sensitivity for detection of anthracycline-induced left ventricular dysfunction in cancer survivors is dramatically increased. ${ }^{59}$ Similarly, in this review, the sensitivity of LVEF, perfusion defects, and global longitudinal strain is increased by cardiac stress tests. ${ }^{16,18,19,22-26}$ This preliminary evidence that cardiac stress tests may be more sensitive for detection of evidence of cardiovascular disease and may also be used to inform the type of heart function tests utilized to address cardiovascular safety in future clinical trials of antineoplastic therapies.

The parameters of cardiac stress tests that were shown to be beneficial in this review were perfusion defects, measured during stress myocardial perfusion imaging, as well as global longitudinal strain reserve and LVCR measured during stress echocardiography. These parameters are associated with negative clinical outcomes. Perfusion defects are reported to be a significant predictor of cardiac events and cardiac death in cancer survivors, and those with reversible perfusion defects (ie, those detected only with stress tests) have a lower probability of 3-year cardiac event-free survival than those with fixed perfusion defects. ${ }^{60}$ Further investigation into the utility of stress myocardial perfusion imaging as a cardiovascular surveillance tool for radiation-treated breast cancer survivors is warranted, given the demonstrated benefit of stress tests in identifying prognostically valuable reversible perfusion defects.

The parameters assessed by stress echocardiography that were beneficial in identifying cardiovascular disease in breast cancer survivors in this review were peak LVEF, ${ }^{16}$ LVCR, ${ }^{16}$ and global longitudinal strain. ${ }^{23}$ Strain imaging is an advanced echocardiographic technique that is new to evaluation with stress. ${ }^{61}$ Strain or strain rate has prognostic and diagnostic value in cardiac disease and surgery, ${ }^{61}$ but its value in the evaluation of anthracycline-induced left ventricular dysfunction is unknown. Additionally, the absence of LVCR (ie, no change or a decrease in LVEF when stress is applied) is associated with decreased myocardial blood flow and oxygen consumption, at rest and under stress, ${ }^{62}$ a maladaptive response predictive of heart failure patient survival. ${ }^{63}$ Although there has been limited research on the use of stress echocardiography for detection of chemotherapy-associated left ventricular dysfunction in breast cancer survivors, the two initial studies ${ }^{16,23}$ highlight the potential for this technique to be used both for prognosis and follow-up in this population.
Future studies are required, investigating the use of sensitive stress echocardiographic parameters, including LVCR, strain, and diastolic function for identification of left ventricular dysfunction associated with anthracyclines and other chemotherapeutic agents.

This review is subject to some limitations. Firstly, no studies involving breast cancer survivors were identified that compared a cardiac stress test to the gold standard for diagnosis of cardiotoxicity, endomyocardial biopsy, which required that a nontraditional method of synthesizing results be developed. Additionally, there was considerable variability in the format of data reported within each study, which made data synthesis difficult. The small number of available studies that fit the inclusion criteria, as well as the small sample sizes ( 8 to 38 ) within these studies limit the interpretation and generalization of the results. Lastly, the findings of this review only provide information as to whether or not cardiac stress tests are beneficial, but do allow for specific recommendations on the optimal timing of this technique with specific breast cancer treatments. However, despite the shortcomings of this review, it demonstrates that there appears to be some benefit to the use of cardiac stress testing of breast cancer survivors to uncover occult cardiovascular disease, and that this technique merits further investigation.

In summary, this is the first review to evaluate the utility of cardiac stress tests in breast cancer survivors. Although few studies of breast cancer survivors have employed cardiac stress tests to monitor cardiac function, and no studies have compared stress tests to the reference standard, the available evidence suggests that myocardial stress may be beneficial in identifying evidence of cardiovascular disease in breast cancer survivors. In particular, stress myocardial perfusion imaging appears to be beneficial in long-term follow-up to left-sided radiotherapy for breast cancer, and stress echocardiography may be beneficial in early and late follow-up to anthracycline treatment. Further research is needed to determine the optimal heart function tests and parameters to be measured during a stress test, the optimal timing of stress tests with different treatments, and the cost-to-benefit ratio of performing a stress test over a heart function test at rest in this population.

\section{Disclosure}

The authors declare that there is no conflict of interest.

\section{References}

1. Bird BR, Swain SM. Cardiac toxicity in breast cancer survivors: Review of potential cardiac problems. Clin Cancer Res. 2008;14(1):14-24.

2. Ewer MS, Glück S. A woman's heart: The impact of adjuvant endocrine therapy on cardiovascular health. Cancer. 2009;115(9):1813-1826. 
3. World Heart Federation. Cardiovascular disease risk factors. Geneva: World Heart Federation; 2013.

4. Minton SE, Munster PN. Chemotherapy-induced amenorrhea and fertility in women undergoing adjuvant treatment for breast cancer. Cancer Control. 2002;9(6):466-472.

5. Riihimaki M, Thomsen H, Brandt A, Sundquist J, Hemminki K. Death causes in breast cancer patients. Ann Oncol. 2012;23(3):604-610.

6. Hooning MJ, Botma A, Aleman BM, et al. Long-term risk of cardiovascular disease in 10-year survivors of breast cancer. J Natl Cancer Inst. 2007;99(5):365-375.

7. Marks LB, Yu X, Prosnitz RG, et al. The incidence and functional consequences of RT-associated cardiac perfusion defects. Int J Radiat Oncol Biol Phys. 2005;63(1):214-223.

8. Jones RL, Swanton C, Ewer MS. Anthracycline cardiotoxicity. Expert Opin Drug Saf. 2006;5(6):791-809.

9. Carver JR, Shapiro CL, Ng A, et al. American Society of Clinical Oncology clinical evidence review on the ongoing care of adult cancer survivors: cardiac and pulmonary late effects. J Clin Oncol 2007;25(25):3991-4008.

10. Dolci A, Dominici R, Cardinale D, Sandri MT, Panteghini M. Biochemical markers for prediction of chemotherapy-induced cardiotoxicity: systematic review of the literature and recommendations for use. Am J Clin Pathol. 2008;130(5):688-695.

11. Fallah-Rad N, Walker JR, Wassef A, et al. The utility of cardiac biomarkers, tissue velocity and strain imaging, and cardiac magnetic resonance imaging in predicting early left ventricular dysfunction in patients with human epidermal growth factor receptor II-positive breast cancer treated with adjuvant trastuzumab therapy. $\mathrm{J} \mathrm{Am} \mathrm{Coll} \mathrm{Cardiol}$. 2011;57(22):2263-2270.

12. Ganz WI, Sridhar KS, Ganz SS, et al. Review of tests for monitoring doxorubicin-induced cardiomyopathy. Oncology. 1996;53(6):461-470

13. Goethals I, De Winter O, De Bondt P, De Sutter J, Dierckx R, Van De Wiele C. The clinical value of nuclear medicine in the assessment of irradiation-induced and anthracycline-associated cardiac damage. Ann Oncol. 2002;13(9):1331-1339.

14. Plana JC. Chemotherapy and the heart. Rev Esp Cardiol (Engl Ed). 2011;64(5):409-415.

15. Manoucheri M, Karunaratne HB. The role of imaging techniques in stress testing. Prim Care. 1994;21(3):535-555.

16. Civelli M, Cardinale D, Martinoni A, et al. Early reduction in left ventricular contractile reserve detected by dobutamine stress echo predicts high-dose chemotherapy-induced cardiac toxicity. Int $J$ Cardiol. 2006;111(1):120-126.

17. Cowen D, Gonzague-Casbianca L, Brenot-Rossi I, et al. Thallium-201 perfusion scintigraphy in the evaluation of late myocardial damage in left-side breast cancer treated with adjuvant radiotherapy. Int $J$ Radiat Oncol Biol Phys. 1998;41(4):809-815.

18. Galluci G, Coccaro M, Storto G, et al. The clinical impact of a cardiologic follow-up in breast cancer aurvivors: an observational study. Int J Immunopathol Pharmacol. 2010;23(4):1221-1227.

19. Gyenes G, Fornander T, Carlens P, Rutqvist LE. Morbidity of ischemic heart disease in early breast cancer 15-20 years after adjuvant radiotherapy. Int J Radiat Oncol Biol Phys. 1994;28(5):1235-1241.

20. Gyenes G, Fornander T, Carlens P, et al. Detection of radiation-induced myocardial damage by technetium- $99 \mathrm{~m}$ sestamibi scintigraphy. Eur $J$ Nucl Med. 1997;24(3):286-292.

21. Haykowsky MJ, Mackey JR, Thompson RB, Jones LW, Paterson DI Adjuvant trastuzumab induces ventricular remodeling despite aerobic exercise training. Clin Cancer Res. 2009;15(15):4963-4967.

22. Højris I, Sand NP, Andersen J, et al. Myocardial perfusion imaging in breast cancer patients treated with or without post-mastectomy radiotherapy. Radiother Oncol. 2000;55(2):163-172.

23. Khouri MG, Hornsby WE, Velazquez EJ, Jones LW, Douglas PS. Exercise stress testing with strain echocardiography is superior to resting echocardiography in identifying doxorubicin-induced preclinical LV dysfunction in breast cancer patients. Circulation. 2011;124:A16399.
24. Seddon B, Cook A, Gothard L, et al. Detection of defects in myocardial perfusion imaging in patients with early breast cancer treated with radiotherapy. Radiother Oncol. 2002;64(1):53-63.

25. Sioka C, Exarchopoulos T, Tasiou I, et al. Myocardial perfusion imaging with $99 \mathrm{mTc}$-tetrofosmin SPECT in breast cancer patients that received postoperative radiotherapy: a case-control study. Radiat Oncol. 2011;6:151.

26. Cassidy J, Merrick MV, Smyth JF, Leonard RC. Cardiotoxicity of mitozantrone assessed by stress and resting nuclear ventriculography. Eur J Cancer Clin Oncol. 1988;24(5):935-938.

27. Lindahl J, Strender LE, Larsson LE, Unsgaard A. Electrocardiographic changes after radiation therapy for carcinoma of the breast. Incidence and functional significance. Acta Radiol Oncol. 1983;22(6):433-440.

28. Kirthi V, Schultz C, Davies SW. Cardiac imaging of trastuzumab-induced cardiomyopathy. Eur J Heart Fail Suppl. 2011;10(Suppl 1):S18.

29. Gustavsson A, Bendahl PO, Cwikiel M, Eskilsson J, Thapper KL, Pahlm O. No serious late cardiac effects after adjuvant radiotherapy following mastectomy in premenopausal women with early breast cancer. Int $J$ Radiat Oncol Biol Phys. 1999;43(4):745-754.

30. Sant M, Allemani C, Capocaccia R, et al. Stage at diagnosis is a key explanation of differences in breast cancer survival across Europe. Int J Cancer. 2003;106(3):416-422.

31. Canadian Cancer Society's Steering Committee on Cancer Statistics. Canadian Cancer Statistics 2012. Toronto: Canadian Cancer Society; 2012.

32. American Cancer Society. Cancer Facts and Figures 2013, 2013 ed. Atlanta: American Cancer Society; 2013.

33. Yeh ET, Tong AT, Lenihan DJ, et al. Cardiovascular complications of cancer therapy: diagnosis, pathogenesis, and management. Circulation. 2004;109(25):3122-3131.

34. Correa CR, Das IJ, Litt HI, et al. Association between tangential beam treatment parameters and cardiac abnormalities after definitive radiation treatment for left-sided breast cancer. Int J Radiat Oncol Biol Phys. 2008;72(2):508-516.

35. Kohli P, Gulati M. Exercise stress testing in women: going back to the basics. Circulation. 2010;122(24):2570-2580.

36. De Wolf D, Suys B, Maurus R, et al. Dobutamine stress echocardiography in the evaluation of late anthracycline cardiotoxicity in childhood cancer survivors. Pediatr Res. 1996;39(3):504-512.

37. Elbl L, Hrstkova H, Chaloupka V, Novotny J, Michalek J. The evaluation of left ventricular function in childhood cancer survivors by pharmacological stress echocardiography. Neoplasma. 2003;50(3):191-197.

38. Guimaraes-Filho FV, Tan DM, Braga JC, Rodrigues A, Waib PH, Matsubara BB. Ventricular systolic reserve in asymptomatic children previously treated with low doses of anthracyclines: a longitudinal, prospective exercise echocardiography study. Pediatr Blood Cancer. 2011;59(3):548-552.

39. Jarfelt M, Kujacic V, Holmgren D, Bjarnason R, Lannering B. Exercise echocardiography reveals subclinical cardiac dysfunction in young adult survivors of childhood acute lymphoblastic leukemia. Pediatr Blood Cancer. 2007;49(6):835-840.

40. Klewer SE, Goldberg SJ, Donnerstein RL, Berg RA, Hutter JJ Jr. Dobutamine stress echocardiography: a sensitive indicator of diminished myocardial function in asymptomatic doxorubicin-treated long-term survivors of childhood cancer. J Am Coll Cardiol. 1992;19(2):394-401.

41. Sieswerda E, Kremer LC, Vidmar S, et al. Exercise echocardiography in asymptomatic survivors of childhood cancer treated with anthracyclines: a prospective follow-up study. Pediatr Blood Cancer. 2010;54(4):579-584.

42. Lanzarini L, Bossi G, Laudisa ML, Klersy C, Aricò M. Lack of clinically significant cardiac dysfunction during intermediate dobutamine doses in long-term childhood cancer survivors exposed to anthracyclines. $\mathrm{Am}$ Heart J. 2000;140(2):315-323.

43. Yildirim A, Sedef Tunaoglu F, Pinarli FG, et al. Early diagnosis of anthracycline toxicity in asymptomatic long-term survivors: dobutamine stress echocardiography and tissue Doppler velocities in normal and abnorma myocardial wall motion. Eur J Echocardiogr. 2010;11(10):814-822. 
44. Beckman JA, Thakore A, Kalinowski BH, Harris JR, Creager MA. Radiation therapy impairs endothelium-dependent vasodilation in humans. J Am Coll Cardiol. 2001;37(3):761-765.

45. Corn BW, Trock BJ, Goodman RL. Irradiation-related ischemic heart disease. J Clin Oncol. 1990;8(4):741-750.

46. Gaya AM, Ashford RF. Cardiac complications of radiation therapy. Clin Oncol (R Coll Radiol). 2005;17(3):153-159.

47. Virmani R, Farb A, Carter AJ, Jones RM. Pathology of radiation-induced coronary artery disease in human and pig. Cardiovasc Radiat Med. 1999;1(1):98-101.

48. Clarke M, Collins R, Darby S, et al. Effects of radiotherapy and of differences in the extent of surgery for early breast cancer on local recurrence and 15-year survival: an overview of the randomised trials. Lancet. 2005;366(9503):2087-2106.

49. Giordano SH, Kuo YF, Freeman JL, Buchholz TA, Hortobagyi GN, Goodwin JS. Risk of cardiac death after adjuvant radiotherapy for breast cancer. J Natl Cancer Inst. 2005;97(6):419-424.

50. Bovelli D, Plataniotis G, Roila F; ESMO Guidelines Working Group. Cardiotoxicity of chemotherapeutic agents and radiotherapy-related heart disease: ESMO Clinical Practice Guidelines. Ann Oncol. 2010; 21 Suppl 5:v277-v282.

51. Bailey IK, Griffith LS, Rouleau J, Strauss W, Pitt B. Thallium-201 myocardial perfusion imaging at rest and during exercise. Comparative sensitivity to electrocardiography in coronary artery disease. Circulation. 1977;55(1):79-87.

52. Hardenbergh PH, Munley MT, Bentel GC, et al. Cardiac perfusion changes in patients treated for breast cancer with radiation therapy and doxorubicin: preliminary results. Int J Radiat Oncol Biol Phys. 2001;49(4):1023-1028.

53. Sicari R, Nihoyannopoulos P, Evangelista A, et al. Stress Echocardiography Expert Consensus Statement - Executive Summary: European Association of Echocardiography (EAE) (a registered branch of the ESC). Eur Heart J. 2009;30(3):278-289.
54. Cerqueira MD. Pharmacologic stress versus maximal-exercise stress for perfusion imaging: which, when, and why? J Nucl Cardiol. 1996; 3(6 Pt 2):S10-S14.

55. Picano E. Economic and biological costs of cardiac imaging. Cardiovasc Ultrasound. 2005;3:13.

56. Marwick TH, Shaw L, Case C, Vasey C, Thomas JD. Clinical and economic impact of exercise electrocardiography and exercise echocardiography in clinical practice. Eur Heart J. 2003;24(12):1153-1163.

57. Chang SM, Nabi F, Xu J, Raza U, Mahmarian JJ. Normal stress-only versus standard stress/rest myocardial perfusion imaging. J Am Coll Cardiol. 2010;55(3):221-230.

58. Ewer MS, Lenihan DJ. Left ventricular ejection fraction and cardiotoxicity: is our ear really to the ground? J Clin Oncol. 2008;26(8):1201-1203.

59. McKillop JH, Bristow MR, Goris ML, Billingham ME, Bockemuehl K. Sensitivity and specificity of radionuclide ejection fractions in doxorubicin cardiotoxicity. Am Heart J. 1983;106(5 Pt 1):1048-1056.

60. Chandra S, Lenihan DJ, Wei W, Yusuf SW, Tong AT. Myocardial perfusion imaging and cardiovascular outcomes in a cancer population. Tex Heart Inst J. 2009;36(3):205-213.

61. Argyle RA, Ray SG. Stress and strain: double trouble or useful tool? Eur J Echocardiogr. 2009;10(6):716-722.

62. Lee HH, Dávila-Román VG, Ludbrook PA, et al. Dependency of contractile reserve on myocardial blood flow: implications for the assessment of myocardial viability with dobutamine stress echocardiography. Circulation. 1997;96(9):2884-2891.

63. Ramahi T. Dobutamine-induced augmentation of left ventricular ejection fraction predicts survival of heart failure patients with severe non-ischaemic cardiomyopathy. Eur Heart J. 2001;22(10):849-856.
International Journal of Women's Health

\section{Publish your work in this journal}

The International Journal of Women's Health is an international, peerreviewed open-access journal publishing original research, reports, editorials, reviews and commentaries on all aspects of women's healthcare including gynecology, obstetrics, and breast cancer. The manuscript management system is completely online and includes

\section{Dovepress}

a very quick and fair peer-review system, which is all easy to use. Visit http://www.dovepress.com/testimonials.php to read real quotes from published authors. 